\title{
Increased mortality among acute respiratory distress patients from immigrant dense urban districts
}

This article was published in the following Dove Medical Press journal: Open Access Emergency Medicine

\author{
Torgny Wessman ${ }^{1,2}$ \\ Rafid Tofik ${ }^{1,2}$ \\ Klas Gränsbo ${ }^{2,3}$ \\ Olle Melander ${ }^{2,3}$
}

'Department of Emergency Medicine, Skåne University Hospital, Malmö, Sweden; ${ }^{2}$ Department of Clinical Sciences, Lund University, Malmö, Sweden; ${ }^{3}$ Department of Internal Medicine, Skåne University Hospital, Malmö, Sweden
Correspondence: Torgny Wessman Department of Emergency Medicine, Skåne University Hospital, Ruth Lundskogs gata 3, SE-20502 Malmö, Sweden

Tel +46 40335712

Mob +46730266445

Fax +46 40336290

Email torgny.wessman@med.lu.se
Purpose: This study investigated whether living in immigrant dense urban districts (IDUDs) and low-income areas in the city of Malmö predicted 5-year mortality among patients admitted to the emergency department (ED) because of acute respiratory distress.

Patients and methods: We randomly selected 184 patients with acute respiratory distress during 2007, visiting the ED at Skåne University Hospital, Malmö. In 2007, Malmö had 36\% first- and second-generation immigrants. The main exposure was defined as being resident in any of the five IDUDs of Malmö compared to being resident in the five districts of Malmö with the highest proportion of Sweden-born inhabitants (SDUDs). We recorded vital parameters; medical triage priority according to Adaptive Process Triage (ADAPT), ICD-10 diagnoses, and the mean annual income for the patient's urban district. We examined 5-year mortality risk using Cox proportional hazards model.

Results: After adjustment for age and gender, patients from IDUDs ( $\mathrm{n}=100,54 \%)$ had an HR $(95 \% \mathrm{CI})$ of $1.65(1.087-2.494 ; P=0.019)$ regarding mortality at 5-year follow-up. Patients in the lowest vs highest income quartile had an HR of $2.00(1.06-3.79 ; P=0.032)$ regarding mortality at 5 -year follow-up. Age, male gender, presence of cardiopulmonary disease, and ADAPT priority also independently predicted the 5-year mortality. The excess risk of 5-year mortality associated with living in IDUDs remained significant after adjustment for age, gender, ADAPT priority, presence of cardiopulmonary disease, and income with an HR of $1.79(1.15-2.78 ; P=0.010)$.

Conclusion: Living in an IDUD is a strong independent risk factor for 5-year mortality in patients with acute respiratory distress. The cause is unknown. Our study suggests a need for better structured follow-up of cardiopulmonary disease in such patients.

Keywords: dyspnea, emergency department, mortality, socioeconomic, income, ADAPT

\section{Introduction}

This study investigated whether living in immigrant dense urban districts (IDUDs) and low-income areas in the city of Malmö predicted 5-year mortality among patients admitted to the emergency department (ED) because of acute respiratory distress. The city of Malmö has a heterogenic population both when it comes to socioeconomic status (SES) and immigrants, with inhabitants from all over the world living in fairly segregated urban districts. It was our interest to see if there are differences in characteristics and mortality for patients with acute respiratory distress visiting the ED at Skåne University Hospital (SUH), Malmö. In 2007, there were a total of $36 \%$ first- and second-generation immigrants (in the following text named as only "immigrants") from 171 countries of the world. ${ }^{1}$ 
Newcomers to the Swedish society from outside of European Union often end up in low SES urban areas and report three to four times as often as Sweden-born people that they suffer from poor or very poor health. ${ }^{2}$ Many diseases that cause chronic respiratory distress depend on SES and lifestyle habits. SES such as low individual education and living in an a low-income area per se is known to be a risk factor for cardiovascular disease (CVD) and all-cause mortality, although some findings are heterogeneous and even absent for men and several CVD diseases..$^{3-5}$

When it comes to population studies from Malmö, significant intraurban differences in mortality from ischemic heart disease have been demonstrated ${ }^{6}$. In addition, immigrants from Iraq and Sweden-born residents in deprived urban districts of Malmö have a higher prevalence of type 2 diabetes. ${ }^{7}$ Both these facts indicate metabolic differences between the groups rather than, for example, differences in blood pressure, which also is a risk factor for ischemic heart disease. Data show that there is an almost linear association between average income and level of immigrants in the urban districts of Malmö. ${ }^{8}$ The lowest average income is seen in the IDUD and vice versa. Patients with respiratory distress are frequently seeking care at EDs. Many different acute and chronic medical conditions cause acute respiratory distress. The most common causes of acute respiratory distress are worsening of chronic diseases in the cardiovascular system and the respiratory system. Many of these conditions have a high mortality.

We hypothesized that differences in lifestyle and socioeconomic and cultural factors linked to living in a low-income area, which also happen to be the IDUD half of Malmö, would translate into increased risk of mortality during 5 years of follow-up in patients with acute respiratory distress.

The aim of this study thus was to test if patients with acute respiratory distress who lived in the low-income and IDUD half of Malmö as compared to those living in the higher income and Sweden-born dense urban district (SDUD) half of Malmö are at an increased risk of death during long-term follow-up.

To address this question, we registered data from the time of ED admission in a cohort seeking care for acute respiratory distress and followed the patients for mortality risk over a median follow-up time of 5.5 years (range 5.0-6.0 years).

The study was approved by the Regional Ethical Review Board of Lund (Dnr 2014/11).

\section{Patients and methods Study population}

During 2007, a total of 63,393 (30,450 females) patients were admitted to the ED at SUH in Malmö.
In all, 4,179 (6.6\%) admissions were caused by acute respiratory distress. When patients arrive to the $\mathrm{ED}$, they are asked for what main problem or symptoms they have at the enrolment desk by a secretary or nurse. Respiratory distress is one of the predefined contact reasons. Of those admitted with acute respiratory distress, 200 patients were randomly extracted to be included in the study. In all, 13 patients were living outside Malmö and thus were excluded. Another three were excluded due to multiple individual visits, leaving us with a cohort of 184 patients. Of the 10 urban districts in Malmö in 2007, we defined the five most immigrant dense districts as IDUDs, whereas the five least immigrant dense districts were defined as SDUDs. In the general population in Malmö, there was a close to linear relationship between average annual income of each district and their proportions of immigrants.

\section{Demography of Malmö}

In 2007, the city of Malmö consisted of 10 different urban districts, some with a high and some with a low frequency of immigrants. The IDUD half of Malmö consisting of the five urban districts with the highest amounts of immigrants had a total of $66 \%$ immigrants with an individual range from $33 \%$ (Kirseberg) to $86 \%$ (Rosengård). The SDUD half of Malmö consisting of the five urban districts with the highest amount of Sweden-born inhabitants had a total of $21 \%$ immigrants with an individual range from $14 \%$ (Västra Innerstaden) to $29 \%$ (Centrum). ${ }^{1}$ Importantly, not only the proportion of immigrants differs between IDUDs and SDUDs but also the country of origin of immigrants. The four most common countries in the IDUD half of Malmö that the first-generation immigrants came from in 2007 were Iraq, Yugoslavia, Bosnia/ Herzegovina, and Denmark, from the highest to the lowest. The four most common countries in the SDUD half of Malmö that the first-generation immigrants came from were Denmark, Poland, Yugoslavia, and Iran.

\section{Exposures}

From the City Council of Malmö, we on a group basis obtained information about the average income of inhabitants in the 1o urban districts. ${ }^{1}$ From the Swedish National Tax Agency (Skatteverket), we received information on an individual basis of the annual income for 2007 for the patients in our cohort. Besides the annual income, we registered the three first diagnoses, priority at arrival, priority after primary survey, heart rate, temperature, systolic and diastolic blood pressures, respiratory rate, oxygen saturation, and date of death. These data were extracted from the patients' medical 
records. We used the ICD-10 to classify diagnoses. In the cohort, 13 visits had a missing principal diagnosis. For 94 visits, there was a second diagnosis, and 52 visits had a third diagnosis. In total, 332 ICD-10 diagnoses were registered in this cohort. At admission to the ED, each patient gets a triage priority by a nurse or a physician according to the severity of his/her medical condition. In 2007, we applied the Adaptive Process Triage (ADAPT) at the ED. ADAPT is a three-level triage system in the process of determining the priority of the patient's treatments based on the severity of his/her symptoms and the severity of his/her vital parameters. ${ }^{9,10}$ This triage system was a forerunner to the medical emergency triage and treatment system (METTS), which was later renamed to the Rapid Emergency Triage Treatment Scale (RETTS), which is used today at EDs nationwide in Sweden. ${ }^{11,12}$ RETTS has four levels of priority according to severity of symptoms and vital parameters, in contrast to the ADAPT system, which has a three-level scoring system. According to the ADAPT system, patients with the highest priority need to see an emergency team of physicians and nurses immediately at arrival, often in the resuscitation room.

\section{Follow-up}

The cohort was observed for mortality during a median follow-up time of 5.5 years (range 5.0-6.0 years), which ended at 31 December 2012. The end point in this study was overall mortality. We used the patient administrative web system (WebPasis) to detect where the patients were living at the moment for extraction of data, ie, when the data were collected from the patients' medical records. It was not possible to see where the patients were living in 2007, which was the time when they actually were visiting the ED. We assumed that relocation of patients from poor urban districts to wealthy urban districts during the period from 2007 to 2012 was not frequent, and even that if patients had moved during that time period, we believe that they may still remain at the same type of socioeconomic neighborhood.

Among the total of 332 diagnoses, there were a total of 94 diagnoses from chapter I in ICD-10, which refers to diseases in the cardiovascular system. There were 97 diagnoses from chapter J in ICD-10, which refers to diseases in the respiratory system. The diagnoses from both chapters (I and J) formed the vast majority of diagnoses for patients with acute respiratory distress in our cohort. We stratified the diagnoses into four categories. Category 1 consisted of admissions with diagnoses from only chapter I. Category 2 consisted of admissions with diagnoses only from chapter J. Category 3 (the double diagnosis group) consisted of admissions with diagnoses from both chapters I and J, which we in the following text call the double diagnoses (DD) group. A fourth category was identified for admissions with diagnoses other than those from chapters I and J.

\section{Statistics}

Data are presented as median (range) or mean $( \pm \mathrm{SD})$, and group-wise differences between continuous variables were compared using independent samples $t$-test or Mann-Whitney $U$ test where appropriate. Categorical variables were compares between groups using the chi-square test. We used Cox regression to relate exposure of IDUDs to mortality in the first model adjusted for age and gender (model 1). The second model was additionally adjusted for annual income (model 2), whereas the third model was additionally adjusted for presence of DD (both cardiovascular and pulmonary diseases diagnosed) and ADAPT priority (model 3). A two-tailed significance level of $(P<0.05)$ was considered as statistically significant.

\section{Results \\ Comparison of baseline characteristics between IDUDs and SDUDs}

Baseline characteristics of IDUD and SDUD patients are given in Table 1. According to the income information on a group level from the city of Malmö, the average income for inhabitants ranged from 78,504 SEK in the poorest district (Rosengård) to 190,172 SEK in the richest district (Västra Innerstaden). The median annual income in 2007 for the patients in our cohort $(\mathrm{N}=184)$ was 133,150 SEK with a range of $0-139,900 \mathrm{SEK}$. As expected, there was a significant difference in median income between patients in the IDUDs, 125,500 SEK (range 0-360,600 SEK), and those in the SDUDs, 140,350 SEK (range 0-1,391,900 SEK; $P=0.016$; Table 1). There was no significant difference in any of the vital signs (pulse, blood pressure, temperature, and oxygen saturation) for the two groups. CVD, respiratory disease, or patients with DD were by far the most common for patients seeking the ED with acute respiratory distress, but the prevalence did not differ between IDUDs and SDUDs. Nor were there any differences in ADAPT priority between IDUDs and SDUDs.

\section{Baseline exposures versus risk of mortality}

There were a total of 94 deaths (51\%) among the 184 patients during the 5-year follow-up time. There was a significant increase in the risk of 5 -year mortality with $65 \%$ of patients 
from IDUDs compared to SDUDs, after adjustment for age and sex (Table 2). There was also a two-fold increase in the risk of 5-year mortality (adjusted for age and sex) in those with an annual income in the lowest quartile (median 45,600 SEK, range 0-81,400 SEK) vs those with an annual income in the highest quartile (median 240,900 SEK, range 201,5001,391,900 SEK; HR=2.0, 95\% CI 1.06-3.79; $P=0.032)$. In addition, whereas patients with CVD or respiratory disease did not have a significantly increased risk of mortality, there was a $76 \%$ increased risk in the age- and sex-adjusted 5-year mortality for patients with double diagnoses, ie, with both respiratory disease and CVD versus those without DD $(\mathrm{HR}=1.76,95 \%$ CI $1.10-2.82 ; P=0.018)$. There was also an increase in the 5 -year mortality risk in $73 \%$ of patients who received the second and third ADAPT triage priority at arrival vs patients with the lowest ADAPT priority $(\mathrm{HR}=1.73,95 \%$ CI $1.10-2.75 ; P=0.019)$. After adjustment of all of these significant variables, on top of age and sex, IDUD vs SDUD remained significantly associated with $79 \%$ increased risk of 5-year mortality ( $P=0.01$; Table 2$)$.

\section{Discussion}

The key finding of our study was that living in the half of Malmö with the lowest annual income and highest density of immigrants (IDUD) was an independent risk factor for increased mortality in patients seeking emergency medical care for acute respiratory distress during a follow-up time of 5 years. Interestingly, the mortality risk linked to IDUDs was independent of several other factors, which also significantly affected survival such as age, male gender, triage priority, yearly income, and having diseases of both the cardiovascular and respiratory system. Compared to some other countries and areas of the world, Sweden and Malmö have relatively low levels of social inequalities. Even so, we find it important

Table I Baseline characteristics of patients admitted to the ED with acute respiratory distress in IDUDs and SDUDs of Malmö

\begin{tabular}{|c|c|c|c|}
\hline Variables & IDUD $(n=100)$ & SDUD $(n=84)$ & $P$-value \\
\hline Age (years) & $65.0 \pm 19.8$ & $67.4 \pm 19.8$ & 0.42 \\
\hline Male gender, $\mathrm{n}(\%)$ & $49(49.0)$ & $44(52.4)$ & 0.65 \\
\hline Median income 2007 (SEK) & $125,500(0-360,600)$ & $\mid 40,350(0-1,39 \mid, 900)$ & $0.016^{a}$ \\
\hline Priority ${ }^{\mathrm{b}} \mathrm{I} / 2 / 3, \mathrm{n}(\%)$ & $9(9.0) / 28(28.0) / 63(63.0)$ & $5(6.0) / 25(29.8) / 54(64.3)$ & 0.73 \\
\hline Heart disease only, n (\%) & $16(16.0)$ & $15(17.9)$ & 0.74 \\
\hline Lung disease only, n (\%) & $28(28.0)$ & $19(22.6)$ & 0.40 \\
\hline $\mathrm{DD}, \mathrm{n}(\%)$ & $15(17.9)$ & $20(20)$ & 0.71 \\
\hline Heart rate $\left(\mathrm{min}^{-1}\right)$ & $91 \pm 20$ & $93 \pm 20$ & 0.68 \\
\hline Body temperature $\left({ }^{\circ} \mathrm{C}\right)$ & $37.4 \pm 0.9$ & $37.3 \pm 0.8$ & 0.54 \\
\hline Systolic BP (mmHg) & $14 \mid \pm 30$ & $140 \pm 27$ & 0.78 \\
\hline Diastolic BP $(\mathrm{mmHg})$ & $77 \pm 16$ & $78 \pm 17$ & 0.81 \\
\hline Respiratory rate $\left(\mathrm{min}^{-1}\right)$ & $23 \pm 8$ & $24 \pm 7$ & 0.90 \\
\hline Oxygen saturation (\%) & $94 \pm 4$ & $94 \pm 6$ & 0.66 \\
\hline
\end{tabular}

Notes: ${ }^{2}$ After Bonferroni correction, there was no significant differences in median income at baseline between IDUDs and SDUDs. ${ }^{\text {bPriority }}$ according to ADAPT. Categorical variables were compared between groups using the chi-square test. DD includes both lung and heart diseases.

Abbreviations: ADAPT, Adaptive Process Triage; BP, blood pressure; DD, double diagnoses; ED, emergency department; IDUD, immigrant dense urban district; SDUD, Sweden-born dense urban district.

Table 2 Patients' 5-year mortality in IDUDs compared to SDUDs

\begin{tabular}{|c|c|c|c|}
\hline & HR & $95 \% \mathrm{Cl}$ & $P$-value \\
\hline \multicolumn{4}{|c|}{ Model I: Adjusted for age and gender } \\
\hline IDUD & 1.65 & $1.09-2.49$ & 0.019 \\
\hline \multicolumn{4}{|c|}{ Model 2: Adjusted for age, gender, and yearly income } \\
\hline IDUD & 1.69 & $1.11-2.56$ & 0.015 \\
\hline Yearly income (QI vs Q4) & 2.00 & $1.06-3.79$ & 0.032 \\
\hline \multicolumn{4}{|c|}{ Model 3: Adjusted for age, gender, yearly income, DD, and ADAPT } \\
\hline IDUD & 1.79 & $1.15-2.78$ & 0.010 \\
\hline Yearly income (QI vs Q4) & 2.27 & $1.18-4.35$ & 0.014 \\
\hline $\mathrm{DD}$ & 1.76 & $1.10-2.82$ & 0.018 \\
\hline ADAPT ( 3 and 2 vs I) & 1.73 & I.10-2.75 & 0.019 \\
\hline
\end{tabular}

Note: DD includes both lung and heart diseases.

Abbreviations: ADAPT, Adaptive Process Triage; DD, double diagnoses; IDUD, immigrant dense urban district; Q, quartile; SDUD, Sweden-born dense urban district. 
to address existing health inequalities, even if it is impossible to counteract factors like origin and difficult to operationalize socioeconomic factors.

Patients from IDUDs have poor SES. With low socioeconomic conditions, independent of origin, may follow differences in diets, physical exercise, low education, employment status, lack of cultural understanding, and lack of social support in the society, which could explain differences in mortality associated with living in IDUDs. Even if our study showed increased mortality in the IDUD half of Malmö even after adjustment for SES, we have to account for that SES per se still could be a stronger factor than immigration status.

Environmental differences within Malmö such as different exposures to pollution and lack of amenities might be possible confounders to different mortality outcomes comparing different neighborhood areas in Malmö. The drinking water in the water tap system for the whole of Malmö comes from the same water supply. Differences between the two halves of Malmö can therefore be assumed to be minor. Studies on the effect of air pollution and some respiratory diseases for the southern Sweden area and Malmö have been made. It was shown that living within $100 \mathrm{~m}$ of a road with $>10$ cars/minute in southern Sweden including Malmö was associated with an increased prevalence of asthma diagnosis and COPD diagnosis, as well as asthma and chronic bronchitis symptoms. ${ }^{13}$ The annual level of $\mathrm{NO}_{2}$ for different socioeconomic groups and countries of birth and educational levels has also been analyzed for the region. However, it was shown that the measured level of air pollution is higher in the center of Malmö rather than that in the suburban areas, where mainly immigrants and inhabitants with lower SES live. Furthermore, it was shown that there is no consistency in how individuals in different socioeconomic classes are exposed to air pollutants. ${ }^{14,15}$ The Swedish Social Welfare System has been diminishing lack of amenities for all inhabitants including immigrants; however, differences in amenities could clearly be a factor explaining differences in mortality. Differences in deaths through violent crime also have to be considered as a confounder comparing different neighborhood areas in Malmö, although less likely to explain differences in mortality in acute respiratory distress patients in our study with a mean age of 65 years.

There are data from other scientific studies showing worse compliance to medication and inequalities in health care services for immigrants. There is evidence that among immigrants from outside the EU25 countries, there is a tendency toward a lesser use of the recommended drugs after myocardial infarction. ${ }^{16}$ However, whether this explains excess mortality in the IDUD group remains speculative.
The implication of this study could be to stronger emphasize existing inequalities in health within Malmö. The study might help to further address such questions as patient-orientated information for immigrants, health education, and language/cultural translators that consider ethnicity and cultural as well as other socioeconomic differences. The society needs to raise issues concerning inequalities when it comes to education, employment, lifestyle habits, and health factors in a socially diverse and multiethnic city like Malmö, even if such factors are difficult to counteract.

Finally, several other factors apart from IDUDs, such as having DD of both cardiac and pulmonary pathologies, as well as high level of prioritization during the transition to emergencies, regardless of IDUD status, are factors of severity and contributed independently to mortality during followup. As these factors are routinely recorded and included in clinical judgment at the ED, the incremental clinical value of adding IDUDs and income in risk prediction and management needs to be studied further. Most importantly, the studied risk factors that remained significant in the multivariate model should not be considered one by one clinically but rather as a summed up burden score.

\section{Limitations}

The relatively small sample size $(\mathrm{N}=184)$ is a limitation of this study, which makes it important to validate our findings in further larger and adjusted studies and evaluate if the results could be replicated. It is a limitation that the collection of data was retrospective, as data were collected after both baseline and follow-up period. We cannot determine whether the relationships shown are casual or not. However, in all observational studies, it is difficult to access causality between exposure variables and outcome. It is also a limitation in this study that we did not have access to individuallevel data on being or not being immigrant. It is a limitation having to generalize to IDUDs and SDUDs. Although having a higher proportion of immigrants, IDUDs are inhabited by several different immigrant groups and native Swedes with lower levels of SES. Another limitation is that we did not have information on smoking habits, educational or occupational status, or other lifestyle habits.

A further limitation is that we in this study could not account for differences in compliance to medication or follow-up within the health care system.

\section{Conclusion}

For patients admitted with acute respiratory distress, living in the half of Malmö with lower SES and IDUDs are strong 
independent risk factors for 5-year mortality. The cause of the increased mortality rates is unknown even if we suspect socioeconomic factors, differences in smoking, and possibly compliance factors rather than pure immigrant status. More and further studies have to pinpoint the underlying mechanisms. Even so, our study suggests a need for better and more structured follow-up of cardiopulmonary disease in such patients.

\section{Ethics approval and consent to participate}

This study has an ethical approval from Regionala Etikprövningsnämnden (EPN), Lund, Sweden Dnr 2014/82. The ethics committee has accepted that consent of participants was not asked for, as data were extracted from patients' charts 5 years after their visit.

\section{Consent for publication}

This manuscript contains no individual person's data and therefore can be published without consent from the participants.

\section{Availability of data and material}

The datasets generated during and/or analyzed during the current study are available from the corresponding author on reasonable request.

\section{Acknowledgments}

The study was supported by research grants from the Knut and Alice Wallenbergs Foundation, Göran Gustafsson Foundation, Swedish Heart-Lung Foundation, Swedish Research Council, Novo Nordisk Foundation, Region Skåne, SUH, and Swedish Foundation for Strategic Research (IRC). Special thanks to Mattias Wieloch, MD, PhD, former section manager, and Maria Ohlson Andersson, MD, present department manager, both at the ED, SUH, Malmö, Sweden, who contributed with support to make this study and paper possible.

\section{Author contributions}

All authors contributed toward data analysis, drafting and revising the paper and agree to be accountable for all aspects of the work. All authors read and approved the final manuscript.

\section{Disclosure}

The authors report no conflicts of interest in this work.

\section{References}

1. Department of Planning, City Executive Office. Official statistics, City of Malmo: 2007.

2. Hjern A. Health in Sweden:The National Public Health Report 2012. Chapter 13, Migration and Public Health. National Board of Health.

3. Pujades-Rodriguez M, Timmis A, Stogiannis D, et al. Socioeconomic deprivation and the incidence of 12 cardiovascular diseases in 1.9 Million Women and men: implications for risk prediction and prevention. PLoS One. 2014;9(8):e104671.

4. Coady SA, Johnson NJ, Hakes JK, Sorlie PD. Individual education, area income, and mortality and recurrence of myocardial infarction in a Medicare cohort: the national longitudinal mortality study. $B M C$ Public Health. 2014;14(1):705.

5. Sorlie PD, Backlund E, Keller JB. Us mortality by economic, demographic, and social characteristics: the national longitudinal mortality study. Am J Public Health. 1995;85(7):949-956.

6. Tydén $P$, Hansen $O$, Janzon L. Intra-urban variations in incidence and mortality in myocardial infarction. Eur Heart J. 1998;19(12):1795-1801.

7. Bennet L, Johansson SE, Agardh CD, et al. High prevalence of type 2 diabetes in Iraqi and Swedish residents in a deprived Swedish neighbourhood - a population based study. BMC Public Health. 2011;11(1):303.

8. The Unit of Planning and Statistics. Official Statistsics, Malmö City Counsil. MalmöNykoSumK5 by Summation Options SuperCROSS. Copyrigt 1993-2012 Space Time Research Pty Ltd. All rights reserved. 9. Nordberg M, Lethvall S, Castrén M. The validity of the triage system ADAPT. Scand J Trauma Resusc Emerg Med. 2010;18(Suppl 1): P36P36.

10. Rasmussen M, Mogensen C. ADAPT as a triage system in a Danish emergency department. Scand J Trauma Resusc Emerg Med. 2012;20(Suppl 2):P3.

11. Widgren BR, Jourak M. Medical emergency Triage and treatment system (METTS): a new protocol in primary triage and secondary priority decision in emergency medicine. J Emerg Med. 2011;40(6): 623-628.

12. Wireklint SC, Elmqvist C, Parenti N, Göransson KE. A descriptive study of registered nurses' application of the triage scale RETTS ${ }^{\circ}$; a Swedish reliability study. Int Emerg Nurs. 2018;38:21-28.

13. Lindrgen A, Stroh E, Montnémery P, et al. Traffic-related air pollution associated with prevalence of asthma and COPD/chronic bronchitis. A cross-sectional study of southern Sweden. Int J Health Geographics. 2009;8:2.

14. Stroh E. The use of GIS in exposure-response studies, a regional study of air pollution and noise in southern Sweden. The Department of Physical Geography an Ecosystem Analysis; Lund University Sweden. Available from: https://www.med.lu.se/content/download/27331/192793/file/ Emilie_Stroh_lic.pdf. Accessed February 18, 2019.

15. Montnémery P, Bengtsson P, Elliot A, Lindholm LH, Nyberg P, Löfdahl CG. Prevalence of obstructive lung diseases and respiratory symptoms in relation to living environment and socio-economic group. Respir Med. 2001;95(9):744-752.

16. Ringbäck Weitoft G, Ericsson Ö, Löfroth E, Rosén M. Equal access to treatment? Population-based follow-up of drugs dispensed to patients after acute myocardial infarction in Sweden. Eur J Clin Pharmacol. 2008;64(4):417-424. 
The Open Access Emergency Medicine is an international, peerreviewed, open access journal publishing original research, reports, editorials, reviews and commentaries on all aspects of emergency medicine. The manuscript management system is completely online and includes a very quick and fair peer-review system, which is all easy to use. Visit http://www.dovepress.com/testimonials.php to read real quotes from published authors.

Submit your manuscript here: https://www.dovepress.com/open-access-emergency-medicine-journal 